\title{
La globalización y su incidencia en la educación superior
}

\author{
Alicia Sequeira Rodríguez
}

\begin{abstract}
Resumen: La globalización es un fenómeno que desafía a la época actual. Unas personas viven fascinadas con sus alcances y lo consideran "inexorable", otras lo piensan como algo natural y muchos tienen más temores que esperanzas frente a los embates que elmismo propicia. En la reflexión siguiente propongo algunas conceptualizaciones de este fenómeno a partir del significado etimológico del término y otras; así como su incidencia en la educación y, a manera de conclusión, propongo algunas reflexiones finales que advierten sobre el papel que debe asumir la Universidad: la investigación sobre este fenómeno y las iniciativas de integración del trabajo que realizan las universidades de la región.
\end{abstract}

La globalización es un fenómeno que desafía a la época actual. Unas personas viven fascinadas con sus alcances y la consideran "inexorable", otras la piensan como algo natural y muchas personas tienen más temores que esperanzas frente a los embates que propicia. En la reflexión siguiente proponemos algunas conceptuaciones de este fenómeno, a partir del significado etimológico del término, así como su incidencia en la educación superior. A manera de conclusión, planteamos algunas reflexiones finales que advierten sobre el papel que debe asumir la Universidad, la investigación sobre la globalización y las iniciativas de integración del trabajo que realizan las universidades tanto localmente como en la región.

\section{El concepto de globalización}

En el presente trabajo, discutimos nueve diferentes conceptos dados al término globalización que, por otra parte, explican la naturaleza y las características de ese fenómeno que necesitamos comprender.

\section{Globalización como: mundialización y universalización}

La palabra "global", de donde viene el término, se refiere al planeta tierra y puede ser sustituida por mundialización y 
universalización, que contiene un sentido de totalidad. James Mittelman (1997), por ejemplo, considera que la globalización es un fenómeno mundial, una fusión de procesos económicos, culturales e ideológicos transnacionales que conduce a que un país penetre en el otro ${ }^{1}$. Readings (1996) afirma que globalización es una clase de "americanización" (americanization), en el sentido de Estados Unidos, que expande su influencia económica por todo el mundo, es decir, por toda la tierra ${ }^{2}$. Este es el mismo sentido que aplica Alejandro Serrano Caldera quien habla de "ética y mundialización", al usar el término mundialización como sinónimo de globalización ${ }^{3}$. Por su parte, Álvaro Montero Mejía (1996) concibe la globalización como: "sinónimo de universalización, aunque el término es empleado para definir la ubicuidad de las noveles relaciones económicas constitutivas de un mercado mundial, supuestamente transparente, fluido, sin fronteras" ${ }^{4}$.

\section{Globalización como homogenización}

Otro significado es sostenido por Robert W. Cox (1997) y pareciera ser el que mejor expresa el proceso de homogenización creciente a nivel económico, social y cultural $^{5}$.

El concepto de globalización, según Cox, como mundialización y universalización supone su propia contradicción porque la mundialización se ve amenazada por las fuerzas de la regionalización y la localización donde se resiste el proceso global. Toda protesta que descuide lo local en aras de lo global, es la expresión de la contradicción del concepto de mundialización. La universalización se ve amenazada por las fuerzas de la particularización. La globalización como proceso de "totalización" implica homogenización pero "olvida" la diferenciación. La fuerza homogeneizante de la globalización genera el concepto de diferencia. Frente a la regionalización, la localización y la diferencia, la globalización como mundialización, universalización y totalización pierde fuerza.

\section{Globalización como fase del sistema capitalista}

La globalización como una fase del desarrollo del sistema capitalista que produce "una extensión mundial de las leyes del mercado"6. Lo global se refiere a ese proceso de expansión del capital que lleva implícito procesos de redistribución de las fuerzas y de los medios de trabajo a merced de los grandes intereses. En esta perspectiva, la globalización incluye la expansión de procedimientos, leyes, modelos de producción, comercio, industria y finanzas en general ${ }^{7}$. Tal como lo señala Ianni (1999), "la globalización del capitalismo es el desarrollo del capital en general, que trasciende mercados y fronteras, regímenes políticos y proyectos nacionales, regionalismos y políticas geográficas, culturas y civilizaciones"8.

La Globalización económica supone centralización de medidas y planes económico-políticos del planeta a merced de un "proteccionismo corporativo", en el cual actúa a favor de las corporaciones que centralizan esa economía y no protege empleos, no favorece comunidades, no fomenta democracias ni preserva la naturaleza ${ }^{9}$. El proceso de globalización crea economías dominadas por el mercado y por la tecnología. El proceso se desprende de aquellas economías con "vocación universal" que evidencian una fuerte contradicción, pues en ellos pocos son los ricos y muchos los pobres. Helio Gallardo afirma que "la globalización comprende una altísima concentración de poder económico"10. 
La conceptuación capitalista de la globalización orienta la organización política con un discurso engañoso, pues se trata de los derechos humanos, la reforma del Estado, la eficiencia, el avance científicotecnológico en el cual el mercado per se traería prosperidad a todo el planeta. Por esta razón, escuchamos hablar, con frecuencia, de modernización del Estado, garantías económicas, reforma educativa, etc. Según Núñez (1996), la globalización alega absoluta supremacía del desarrollo económico, existencia de un "mercado libre" sin regulación, destrucción de los modelos económicos de "sustitución de importaciones" -que originan autosuficiencia- incremento de economías orientadas hacia la exportación, privatización de la empresa pública y promoción excesiva del consumo ${ }^{11}$.

La conceptuación capitalista de globalización necesita de las clases subordinadas, para mantenerse; en ellas se fundamenta su supervivencia. La globalización es el avance del capitalismo que, con el conocimiento, el prestigio y los recursos presentes, expande en las clases subordinadas del resto del mundo su contradicción. Por esto el capitalismo no se preocupa porque estos sectores, en esta situación de subordinación dejen de ser subordinados, porque ellos son la fuente de explotación social que se incrementa con aranceles favorables, deterioro de los empleos o las garantías económicas lo que no debe confundirse con garantías sociales para la mayoría del pueblo.

La conceptuación capitalista de globalización destaca el modelo democrático neoliberal, la concentración del capital corresponde al carácter de la nueva revolución donde esa acumulación depende cada vez más de la intensidad de los recursos naturales y del trabajo, e incluso de la intensidad del capital para concentrarse en una "acumulación tecnológica basada en la intensi- dad del conocimiento". Por esta razón, se está de acuerdo cuando se afirma que "la concentración y centralización del conocimiento tecnológico es más intensa y monopólica que las otras formas de capital, aumentando la brecha entre el Norte y el Sur"12.

\section{Globalización como proceso neoliberal}

El neoliberalismo es un modelo basado en el paradigma cartesiano-newtoniano de los siglos XVII-XIX (Capra, $1985)^{13}$, modelo positivo, de enfoque lineal, reduccionista y fragmentario, con carácter analítico más que sintético. Según Orellana Rosal (1997), el neoliberalismo es el intento de:

“...modelar la civilización del futuro, de acuerdo con la teoría económica y política del Siglo de las Luces y la ilustración europea de los albores de la Revolución Industrial y la modernidad"14.

Fernando Rojas (1991), se pregunta ¿qué es? o ¿qué no es? este fenómeno llamado neoliberalismo y afirma que "no es una mera doctrina económica" sino más bien "una constelación estratégica [globalización] para la renovación del modelo de producción capitalista en el mundo"15. Por otra parte, Perry Anderson (1996), citado por Ezcurra (1998), afirma que "el neoliberalismo surgió después de la Segunda Guerra Mundial como una 'reacción teórica y política vehemente' contra el Estado de Bienestar"16, que logra su mayor extensión en la década de los años ochenta con su hegemonía a escala mundial y que, como afirma Hinkelammert (1996), dice que provoca crisis pero no está en crisis, sino que al contrario, "florece como nunca"17.

El neoliberalismo promueve la exportación y la política de liberalización del mercado. Citando a Pedro Vuskovic (1994) 
y refiriéndose a las características del neoliberalismo, Ezcurra dice:

“Sus 'ejes básicos' se pueden resumir en: a) '...constituir a las exportaciones en la fuente fundamental del crecimiento...' lo que lleva a políticas como el estímulo de 'aperturas incondicionales' al capital transnacional y el sobreacento en la 'competitividad'; b) 'reducir drásticamente el ámbito de acción del Estado y propiciar la privatización de toda suerte de actividades productivas y servicios...'; c) y respecto del corto plazo, jerarquizar por encima de cualquier otro objetivo la preservación de los 'equilibrios macroeco-nómicos' (sobre todo, en materia de presiones inflacionarias y de las cuentas fiscales y externas). Precisamente, tales 'ejes' definen a los 'ajustes' que, por ende, no se limitan a la búsqueda de 'equilibrios macroeconómicos', sino que también impelen transformaciones 'estructurales' (reforma externa y del Estado)"18.

El neoliberalismo considera el mercado como el mejor asignador de los recursos económicos y el mejor distribuidor del ingreso, en el marco de la noción de equilibrio automático (espontáneo y natural) derivado del modelo newtoniano de un universo en equilibrio perfecto. El modelo neoliberal propone que la actividad económica debe pasar del Estado a la sociedad civil, ya sea por inversión de capitales nacionales o transnacionales. Será la ideología de libre competencia, el premio a los mejor adaptados, los más egocéntricos, agresivos y violentos, los que seguirán promoviendo los juegos de guerra con fines de control y dominio mundiales, juegos que se acomodan a los intereses de las transnacionales de la civilización industrial y de los círculos gobernantes vinculados a esos intereses ${ }^{19}$.

Promueve "una democratización global", un paradigma universal, mundial, integral y planetario. Este paradigma fue impulsado por el Ex-presidente Ronald Reagan, seguido por el Ex-presidente Bush y, por la administración Clinton con su "alianza global para la democracia". "Se trata -como afirma Ezcurra- de "un proyecto político formulado, lanzado y soste- nido por los Estados del capitalismo avanzado"$^{20}$. Así, Gorostiaga (2000) dice que:

"La economía se convirtió en el eje dominante de las relaciones entre el Estado, Mercado y Sociedad Civil. Los bancos y las empresas transnacionales especialmente con sus gigantescas fusiones ("mergers") crearon un liderazgo empresarial de las grandes corporaciones y conglomerados económicos que comenzó a hegemonizar en forma creciente a los partidos y al propio Estado, cooptando en buena parte a sectores importantes de la sociedad civil"21.

No obstante, el modelo neoliberal tiene también su contradicción en la crítica del paradigma cartesiano newtoniano, crítica que se extiende al modelo positivista, a los enfoques lineales, al reduccionismo y fragmentarismo y al criterio empírico-analítico que alega superioridad científica. Lo mismo pasa con la contradicción que generan las aperturas al capital transnacional y la competitividad, la reducción de la acción del Estado y el proceso de privatización, la importancia del equilibrio espontáneo y especialmente el macroeconómico, la importancia de la libre competencia, la lógica del mercado y la invasión de capitales transnacionales. Una homogenización que violenta las dinámicas de la economía local, de la organización social vigentes, de las políticas autóctonas y la identidad de lo particular. Razón por la cual se generan protestas, marchas, rebeliones, inclusive armadas.

\section{Globalización como concepción triunfalista}

Esta concepción se da en el contexto donde el socialismo era considerado, en sus momentos de avance, una amenaza para el capitalismo, amenaza que, a la altura de estos tiempos (el año 2000), algunos dan por superada. Se considera al socialismo como desviación de la globalización capitalista y obviamente como amenaza alternativa. El 
paradigma socialista, representado por los países socialistas (Europa del Este) debía ser destruido. Sin embargo, el avance de ese socialismo fue interrumpido con el derrumbe del mundo comunista considerado, como dice Helio Gallardo, "la muerte de Marx, de Lenin, de la URSS, de China, de Cuba, del socialismo, el marxismo y del comunismo, y con ellos, de la idea y de la práctica de la revolución social"22.

El socialismo es la contradicción del capitalismo. Por eso, Gallardo continúa afirmando

“...la crisis del socialismo histórico no puede traducirse sino como triunfo del capitalismo -muchas veces, un triunfo total, metafísico-, con entera independencia de la historicidad de representaciones como 'economía centralmente planificada' y 'economía de mercado' y sus relaciones, también históricas, y, sobre todo, sin ninguna preocupación por el alcance o sentido material de la expresión 'triunfo del capitalismo', que implica la muerte de la utopía"23.

El concepto triunfalista de la globalización genera su propia contradicción, que ayer pudo ser el socialismo -que aún despierta lástima, desprecio o represión- y que hoy podría ser cualquier otra alternativa o la simple resistencia que amenace la "seguridad nacional". Esto se debe a que el socialismo subyace como posible alternativa para el futuro aunque con nuevas estrategias y tácticas; inclusive podría incorporar el cooperativismo y el comunitarismo. El capitalismo generó -como dice Cox- un "Corto Siglo XX" (1914-1991) de socialismo real; lo que no niega es su actual fuerza subyacente.

\section{Globalización como economía}

La economía global que ya tiene larga existencia entre nosotros, distingue entre economía internacional y economía mundial. La primera es aquella de movimientos comerciales, inversiones, pagos internacionales, normada por los estados y las organizaciones internacionales creadas para ese propósito. La segunda es la economía -producción y finanzas organizadas en redes (networks) - existente más allá del poder normativo nacional e internacional. Esta doble comprensión coincidió con una política económica de reestructuración de la producción transnacional masiva de bienes "estandarizados", el consumo de menos energía, nuevos métodos de trabajo y la concentración del conocimiento científico. El ataque al modelo económico "fordista"24 y la reducción del presupuesto estatal -especialmente para la política social-, eliminaron regulaciones, incrementaron los procesos de privatización y la competencia internacional. Además, no sólo avanzó en la tecnología sino que también se aplicó a la producción y a la comunicación, para mencionar los campos más importantes de su aplicación.

Esta perspectiva se fundamenta en un concepto de tiempo espacial, uniforme y homogéneo, genera una ideología cuantitativa que sacrifica lo cualitativo, considera la realidad estática, hecha en forma definitiva y donde el futuro es fácilmente predecible. Este concepto orienta en las relaciones complejas del presente, donde éste es fijo y determinado y donde la globalización no sólo es algo necesario sino definitivo; el futuro es imaginable sólo como el posterior desarrollo de las tendencias del presente, sin creación de nada nuevo.

La globalización económica lleva la recesión como contradicción que no sólo afecta a las economías desarrolladas (1973) sino, de una manera intensa, a los países subdesarrollados. La crisis se generalizó al final de la década de los años setenta.

Desde esta perspectiva, Quesada Mateo citando a Mark Ritchie Prendale del Instituto Agrícola y Política de Comercio Norteamericano, dice que: 
"La globalización es un proceso económico, en el cual las grandes corporaciones mueven dinero, fábricas y bienes alrededor del planeta a cada vez mayor velocidad en busca de mano de obra y materias primas más baratas además de una débil protección al trabajador y al ambiente. La globalización es una ideología que asume que los seres humanos y el planeta estarán mejor si el mercado global se separa de las consideraciones éticas, sociales y ambientales" 25 .

Esto hace más complejas las contradicciones del nuevo modelo económico pues la globalización "tiende a convertir al mundo en un campo propiedad de pocos siguiendo el modelo que aplicó el Imperio Romano en todo el territorio entonces bajo su dominio"26.

\section{Globalización como "lógica de mercado"}

Este concepto crea una nueva situación para el mercado y sus agentes. La globalización se presenta como finalidad, como culminación inevitable de las tendencias poderosas del mercado sobre el trabajo. El dominio de esas fuerzas económicas son consideradas como necesarias y beneficiosas. Los Estados y los sistemas interestatales deben servir para asegurar esa lógica; sus principales agentes ideológicos son las corporaciones multinacionales y los bancos que proponen el nuevo modelo del así llamado desarrollo económico. Lo inevitable de la lógica es que no permite otra alternativa.

Esa lógica tiene su propia contradicción en los pueblos ricos y pobres que adoptan nuevas políticas y las mayorías son relegadas fuera de la esfera de la economía mundial. La globalización necesita una política de represión y de fuerza militar: se previene la desestabilización de la economía mundial que podría venir de la protesta por las desventajas de los que se quedan fuera de esa economía. La nueva lógica obliga al retorno a una política de "seguridad nacional" que, con nuevos nombres y nuevas formas, interpreta como "seguridad" y "nacionalidad" la vigencia de ese "nuevo orden" en todo el mundo y en beneficio del país o países que globalizan.

\section{Globalización como ideología}

Este concepto justifica la economía internacional y mundial. Dice Cox que esta economía:

“...creció aprovechándose de la fragmentación de la economía internacional, lo que permite al capital escoger los lugares más propicios en los cuales ubica las diversas fases de una geografía diseminada del proceso de producción, tomando en cuenta las diferencias en los costos laborales, las regulaciones ambientales, los incentivos fiscales, la estabilidad política y otros factores" 27 .

Esto es lo que permite al capital manejar sus cuentas de manera que sus ganancias podrían adquirirse donde los bajos impuestos prevalecen.

Son los intereses creados los que convierten la globalización en "aparato ideológico" 28 de las clases adineradas que por medio de corporaciones, banca y gobiernos, se expande y hace creer que ella es opción inevitable e impulsa a miles de invadidos por esa globalización -además de explotados y oprimidos- a defenderla y a trabajar por ella, alegando que ha llegado el "fin de las ideologías".

$\mathrm{Su}$ contradicción consiste en que el fin de las ideologías que alegan se basa en que el socialismo real -la ideología alternativa que la amenazaba- fracasó. Si esto es históricamente cierto, por supuesto que el fin de la ideología socialista habrá cavado su sepultura. Pero esto no quiere decir que no haya otras ideologías vigentes, que la ideología del socialismo ideal no tenga vigencia o que la misma globalización no sea una ideología. Al contrario, el capitalismo, 
el socialismo ideal y las ideologías alternativas pueblan el universo actual. Carlos Fuentes se refiere a una de ellas cuando afirma que:

"La globalización no es un destino manifiesto... Nuestros países no deben abrirse a la globalización sino garantizar previamente el respeto a la soberanía y mejorar sus condiciones internas. La globalización debe mejorar las condiciones de vida de todos los países, especialmente de los más débiles, potenciar la diversidad como recurso de fuente inagotable de ideas y progreso pero no como "destino manifiesto"29.

No es que hayan muerto las ideologías ni que haya una sola ideología de la globalización sino que lo que no ha muerto son las actuales condiciones de vida de los países latinoamericanos, de los países africanos, de los países asiáticos, de los más débiles, generadoras de ideologías alternativas a la globalización como ideología. Montero Mejía dice que:

"Los países centrales, los que han alcanzado el mayor desarrollo económico, no sólo son capaces de dictar las normas de las relaciones económicas internacionales, sino que acompañan esos dictados y condiciones con una visión particular del mundo. Han impuesto globalmente una ideología que, compartida por casi todos los países, les garantiza que la humanidad viva de acuerdo con sus intereses y acepte, como las mejores posibles, las relaciones políticas económicas fijadas por ellas. Así se globalizan las reglas del juego económico y se globalizan las formas de pensar" 30 .

\section{Globalización como perspectiva anti-ecológica}

Este concepto ve sus implicaciones en el planeta (globalización) y en la organización de la producción (globalismo), especialmente en las condiciones de vida de las tres últimas décadas del siglo XX. La globalización ha creado evidentes contradicciones en las clases subordinadas de los países capitalistas y no capitalistas; ella ha generado una producción que deteriora el planeta y generaliza contaminación, afecta la naturaleza, amplía la separación de las condiciones de vida entre el pequeño segmento integrado a la población global -la red financiera- y las condiciones del resto del mundo.

Por esta razón, no es lo mismo hablar del bienestar de las naciones industrializadas que de la situación de los países pobres. El globalismo plantea una pregunta ética fundamental en torno a las condiciones donde el rico se apropia de los recursos del mundo y los deteriora y donde se frustran más las aspiraciones de los pobres por adquirir un nivel de vida digno. Una naturaleza contaminada achica las expectativas de esas aspiraciones.

La perspectiva ecológica de la globalización surge a mitad de la década de los años setenta y tiene que ver con el papel que han jugado los estados en la extensión de la competencia económica y la explotación de la naturaleza. Ese papel ha traído consecuencias en dos sentidos: el planetario y el de la organización de la vida humana y del trabajo. Pareciera que la consigna hubiera sido "pensar globalmente y actuar localmente", los que globalizan son los que piensan y los globalizados son los que actúan, los pocos son los que tienen y deciden y los muchos son los que no tienen y obedecen.

\section{La incidencia de la globalización en la educación superior}

Estamos de acuerdo con afirmar que la globalización es un fenómeno de trascendencia, de gran intensidad y aceleración que trasciende el campo económico cuya caracterización Tünnerman (1999) plantea al afirmar que: "globalización no se limita al aspecto puramente económico; en realidad, es un proceso multidimensional que comprende aspectos vinculados a la economía, las finanzas, la ciencia y la tecnología, las comunicaciones, la cultura, la política, etc... ${ }^{31} \mathrm{~A}$ continuación analizaremos algunos 
aspectos en los cuales se nota la incidencia de la globalización en la Educación Superior y que, a lo mejor, no estamos tan conscientes de que tenemos que hacer una valoración seria y en profundidad de nuestro quehacer académico. La pregunta básica es ¿de qué manera responde la Universidad a las demandas de un mundo en el cuál, los países en desarrollo son globalizados pero no tienen ni a largo, mediano o corto plazo capacidad para globalizar?

En concreto, los aspectos que inciden son los siguientes:

1. Vivimos una nueva época en la cuál, el proceso de transición entre lo que fue la revolución industrial y la revolución posindustrial nos lleva a adentrarnos en la tercera revolución de la humanidad en la cual el desarrollo científico y tecnológico demanda del trabajo universitario un "aggiornamento", esto es, una puesta al día en todos los ámbitos de su quehacer académico. A este respecto, Tünnerman (1999) nos dice: "Internet ya es el siglo XXI, la protección del genoma humano, ya es el siglo XXI, la expansión creciente de las "culturas híbridas", la irrupción de las "culturas virtuales" ya es el siglo XXI, la educación permanente, ya es el siglo XXI: y la revolución ecológica y energética, ya es el siglo XXI"32. Surge lo que algunos llaman la 'sociedad del conocimiento', sociedad de la información o de la era de la informática. El desarrollo de las comunicaciones, del transporte y de la tecnología que contribuyen a flexibilizar los procesos de trabajo agregan nuevos problemas al mundo del trabajo como es, por ejemplo, la automatización. La robótica se impulsa en una cantidad de campos como la micromecánica, la medicina, en la industria, en general. Las redes de comunicación me- diante el empleo de las computadoras cubren todo el globo y su impacto ya empieza a hacerse evidente en la transformación del modo de vivir $\mathrm{y}$, sobre todo, del modo de trabajar (Cf. Devandas:1998:6), los cambios en la división social del trabajo, en los aspectos técnicos, sociales e internacionales, el aprovechamiento del bajo costo de la mano de obra en las regiones nuestras, sobre todo, la tendencia a la "feminización del empleo"33. Estos son aspectos que están retando a la Educación Superior. La Universidad se ve obligada a responder a los nuevos desafíos que le presenta el mundo globalizado.

2. En los procesos educativos, algunas instituciones se han visto precisadas a "violentar" sus procesos pedagógicos y administrativos para responder a las demandas de las compañías trasnacionales. Se han dislocado las normativas de admisión de estudiantes en algunas instituciones, se han importado planes de estudio de países desarrollados para ponerlos en funcionamiento en otro contexto, el nuestro, por ejemplo, sin ningún diagnóstico, sin ningún pronóstico, sin ninguna programación y sin ninguna evaluación previa. Todo se deja al entusiasmo y sumisión de unos pocos que responden en nombre de toda una comunidad universitaria y del país.

3. En lo económico también se nota la incidencia de la globalización puesto que la educación ha dejado de ser prioridad para algunos estados, como por ejemplo, el estado costarricense. El gasto educativo ha bajado, a partir de la década de los 80 s y se ha tenido complacencia estatal en relación con la apertura de la educación superior privada que ya pasan, en Costa Rica, de 40 instituciones y algunas de ellas 
no reúnen los requisitos académicos para su funcionamiento.

4. Los salarios que se ofrecen a los docentes de la Educación Superior no compiten con la empresa y eso hace que se dé "fuga de cerebros" y la Universidad no pueda beneficiarse del aporte de sus mismos graduados y becarios.

Podríamos seguir mencionado otros aspectos de la globalización que inciden en la Educación Superior. Dejaremos para que los participantes y las participantes en este I Simposio Internacional de Pedagogía Universitaria contribuyan con su experiencia a aumentar esta lista. Ahora, tracemos algunas líneas, a manera de reflexión final de este trabajo que podrían contribuir, de manera que la Universidad fortalezca su presencia crítica, lúcida y creativa en la sociedad en la cual vivimos:

1. Es imperativo que la Universidad defina, de una vez por todas, su enfoque educativo de manera que se aparte de ese modelo mecánico, vertical, academicista que domina el quehacer académico universitario. La universidad está llamada a hacer ciencia, a apuntar todas sus "baterias" a plantear un paradigma pedagógico que le permita ser voz anunciadora de una sociedad con equidad, una sociedad que propicie la justicia, una sociedad que lucha por la paz. En este sentido, la Universidad tendría que asumir un enfoque dialéctico que propicie una orientación inclusiva en el cual el aporte cuantitativo tiene lugar pero, sobre todo, encamine su trabajo científico a una dimensión cualitativa de entrega $\mathrm{y}$ compromiso por los excluidos.

2. Aunar esfuerzos de integración. Hoy día se habla de procesos de acreditación, de autoevaluación, de calidad total. Cualquier persona podría pensar que esos procesos nos llevan a un compartir equitativo, a una integración. Si bien es cierto, la globalización ha permito una mayor comunicación entre los seres humanos lo que predomina es la imposición de los globalizadores a los globalizados. Se requiere, como bien se afirma, gobernar la globalización y esta es una responsabilidad compartida y la Universidad podría aportar integrando su trabajo tanto en su propia institución como a nivel interuniversitario y a nivel latinoamericano. Utilizar los recursos al alcance para mejorar los canales de comunicación, creando redes, investigaciones paralelas entre nuestras universidades y otras universidades del ámbito latinoamericano, intercambiando docentes y estudiantes y, sobre todo, investigando las coyunturas que viven nuestros pueblos de manera que podamos construir nuestro propio paradigma, nuestro propio modelo de desarrollo y nuestro propio proceso educativo.

Dos desafíos a los cuales las Universidades deberían responder. Esto sólo será posible con el compromiso que la misión universitaria impone al servicio del país donde trabaja.

\section{Notas}

1. Cf. James H. Mittelman (1997 ed.). Pp. 1-19. Véase el aporte de Rodrigo Quesada Monge (1998). Pp. 25-28 y de Alvin y Heidi Toffler (Noviembre 1, 1998) p. 16A .

2. B. Readings 1996. P. 35.

3. Alejandro Serrano Caldera, 1995.

4. Álvaro Montero Mejía, (1996) P. 31.Consultar también José, R. Villalón, S. Th.D. (1998). Pp. 3-8. 
5. Robert W. Cox, 1997. Pp. 21-30. Consúltese también Javier Mendoza Rojas, 1995. P. 103, José Pablo Cob Barboza, 1998.

6. Luis Guillermo Herrera, 1995. P. 7.

7. Cf. Alicia Sequeira, agosto, 1998. P. 1. Consúltese también José Joaquín Brumner, p. 9, a Carlos Tünnerman Bernheim, 1999. P. 62, Octavio Ianni, 1998. P. 85.

8. Ostavio Ianni, 1999. P. 16.

9. $\quad C f$. A. Núñez (s.f.). P. 13.

10. Helio Gallardo (s.f.) P. 17. Consultar también a Enrique Iglesias, mayo 22, 2000). P. 6 A y a Michael Candessus, p. 6A.

11. Cf. Núñez. Op. Cit. P. 13.

12. Xavier Gorostiaga. Op. Cit. P. 3.

13. Cf. Fritjof Capra, 1982. Pp. 55-79.

14. César Orellana Rosal, 1997 . P. 109.

15. $\quad C f$. Fernando Rojas H., Julio, 1991. P. 1.

16. Ana M. Ezcurra, Jan/jun, 1998. P. 92.

17. Ibid. P. 94. Cf. también en Franz Hinkelammert, 1996.

18. Ana María Ezcurra. Op. Cit. Pp. 93 y 94.

19. Cf. Revista ABRA 25-26, Facultad de Ciencias Sociales, Heredia. P. 110.

20. Ana M. Ezcurra. Op. Cit. P. 96.

21. Xabier Gorostiaga S.J, Febrero 23-26, 2000. P. 8.

22. Helio Gallardo, 1991. P. 155.

23. Ibid.

24. Dice Stewart R. Clegg que "las organizaciones modernas pueden ser pensadas en términos de la tipificación de Weber de las estructuras mecánicas burocratizadas de control, como estas fueron subsecuentemente levantadas sobre una base totalmente racionalizada del trabajo dividido y sin pericia. Esos fundamentos son generalmente referidos como 'fordismo'". El fordismo era "un sistema de producción en masa basado en ambos, el incremento productivo del trabajo y su relación con el salario que ligaba el salario real con el crecimiento productivo creciente..." ("organizations may be thought of in terms of Weber's typification of bureaucratized, mechanistic structures of control, as these were subsequently erected upon a fully rationalized base of divides and deskilled labour... These foundations are usually referred to as those of 'Fordism'". "A system of mass production based on both the increases in labour productivity and the wage relation which linked real wage and productivity growth..."). Stewart R. Clegg. Consultar también Leonard Mertens (1990).

25. Op. Cit. Quesada Mateo. P. 21.

26. Rodrigo Carazo Odio, mayo 1997. P. 32.

27. Ibid. P. 23.

28. Término usado por Louis Althusser, 1975. Pp. 107-172.

29. Manuel Bermúdez, Julio, 1997. P. 12.

30. Álvaro Montero Mejía. Op. Cit. P. 32.

31. Carlos Tünnerman B., 1999. P. 62.

32. Ibid. P. 58.

33. Cf. Mario Devandas Brenes, agosto, 1998.

\section{Referencias bibliográficas}

Althusser, Louis. "Sobre la Ideología y el Estado". En: Escritos 1968-1970 de Louis Althusser. Barcelona: Editorial Laía, pp. 107-172. 1975.

Bermúdez, Manuel. Entrevista realizada al escritor mexicano Carlos Fuentes. Semanario Universidad. Universidad de Costa Rica. San José, Costa Rica, p. 12. Julio, 1997.

Brumner, José Joaquín. Educación: Escenario del futuro. Nuevas tecnologías $y$ sociedad de la información. PREAL: Chile, p. 9. Enero, 2000. 
Capra, Fritjof. El Punto Crucial. Argentina: Editorial Troquel, S.A. pp. 5579. 1982.

Carazo Odio, Rodrigo. Exposición del Lic. Rodrigo Carazo Odio. En: Foro. Retos del desarrollo sostenible dentro del contexto de globalización. Memoria. Consejo Nacional de Rectores, Oficina de Planificación de la Educación Superior, p. 32. Mayo, 1997.

Clegg, Stewart R. "New Organizational Forms." En: Managing Strategic Action, Mobilizing Change, Concepts, Readings and Cases, by Cynthia Hardy. (s.a.).

Cob Barboza, José Pablo. El concepto de América Latina en el sistema de educación formal costarricense. Tesis de grado, Licenciatura en Estudios Latinoamericanos, Facultad de Filosofía y Letras, Instituto de Estudios Latinoamericanos, Universidad Nacional, Campus Omar Dengo, Heredia, Costa Rica. 1998.

Cox, Robert W. "A perspective on Globalization". En: Globalización: Critical Reflections. James H. Mittelman, ed. Boulder, London: Lynne Reinner Publishers, pp. 21-30. 1997.

Devandas Brenes, Mario. Globalización, Integración y equidad: El caso de Costa Rica. San José, Costa Rica: Universidad Estatal a Distancia. Agosto, 1998.

Escurra, Ana M. Globalización, neoliberalismo y sociedad civil. Algunos desafíos para los movimientos sociales y populares latinoamericanos. En: Revista Educacao \& Filosofia de la Universidad Federal de Uberlandia de Brasil, v. 12. n. 23, 89-127: p. 92. Jan./Jun., 1998.
Fonseca González, Vanesa. "Exorcisando al fantasma de la cultura global, identidad nacional y globalización”. En: Revista de Ciencias Sociales, Número 82, Universidad de Costa Rica, p. 85. Diciembre, 1998.

Gallardo, Helio. Crisis del socialismo histórico, ideologías y desafíos. San José, Costa Rica: Departamento Ecuménico de Investigaciones, p. 155. 1991.

. Reforma política, gobierno, Estado. Semanario Universidad. San José, Costa Rica. p. 17. (s.f.).

Habermas, Jürgem. "Conocimiento e interés". En: Ciencia y Técnica como "ideología". Madrid: Editorial Tecnos, S. A., pp. 159-181. 1989.

Herrera, Luis Guillermo. La Globalización. Semanario Universidad, p. 7. San José, Costa Rica. 1995.

Hinkelammert, Franz. El mapa del emperador. Determinismo, caos, sujeto. San José, C.R.: DEI. 1996.

Ianni, Octavio. Teorías de la globalización. México: Siglo XXI Editores. 1998.

. La era del globalismo. Madrid: Siglo Veintiuno de España, Editores, S.A. p. 16. 1999.

Iglesias, Enrique. Entrevista por Lidieth Brenes, Redactora del periódico $L a$ Nación. Enrique Iglesias es el Presidente del Banco Internacional de Desarrollo (BID), p. 6A, mayo 22, 2000.

Mendoza Rojas, Javier. "La universidad frente a las tendencias de la globalización”. México: UNAM. En: Escenarios para la Universidad contemporánea, p. 103. 1995. 
Mertens, Leonard. Crisis económica y revolución tecnológica. Hacia nuevas estrategias de organización sindical: Venezuela: Editorial Nueva Sociedad. 1990.

Michael Candessus, Discurso antes de dejar la dirección del Fondo Monetario Internacional (FMI). (Boletín del FNI FMI, 28 de febrero 2000). La Nación, Mayo 22 de 2000. P. 6A.

Mittelman, James H. "The Dynamics of Globalization" En: Globalization: Critical Reflections London: Lynne Reinner Publishers, pp. 1-19. 1997.

Montero Mejía, Álvaro. El mundo desigual. Ensayos frente al neoliberalismo. San José, Costa Rica: Editorial EUNED, p. 31. 1996.

Núñez, A. Globalización y Neoliberalismo. Dos palabrotas de la política contemporánea. Periódico Universitario Diálogo. San Juan, Puerto Rico. p. 13. (s.f.).

Orellana Rosal, César. "Costa Rica, hacia el futuro". En Revista de la Facultad de Ciencias Sociales ABRA, 25-26, I y II Semes-tres 1997, Universidad Nacional: EUNA, Heredia, Costa Rica, p. 109. 1997.

Quesada Monge, Rodrigo. Globalización y deshumanización: dos caras del capitalismo avanzado. 1er. ed. Heredia, Costa Rica: EUNA, p. 25-28. 1998.

R. Villalón, S., José Th.D. “Globalización, un término en busca de una definición." Puerto Rico: Organización para el Fomento del Desarrollo del Pensamiento Inc. En: Crecemos. Revista de Desarrollo Humano y Pensamiento, Año 4, Número 1, pp. 3-8. 1998.
Readings, B. The University in Ruins. Cambridge: Massachusetts and London, p. 35. 1996.

Revista ABRA 25-26, Facultad de Ciencias Sociales, Heredia, p. 110. (s. a.).

Rojas H., Fernando. "Neoliberalimo ¿Qué es? ¿Qué no es?”. En: CRIE. México: Centro Regional de Informaciones Ecuménicas, n. 275, p. 1. Julio, 1991.

Sequeira, Alicia. América Latina frente a los procesos de Globalización. Trabajo presentado en la actividad académica coordinada por la Dra. Marielos Giralt B. Doctorado en Educación, Facultad de Educación, Universidad de Costa Rica, p. 1. Agosto, 1998.

Serrano Caldera, Alejandro. Ética y mundialización Ponencia al II Congreso Mesoamericano de filosofía. Universidad Centroamericana, Managua, Nicaragua. Diciembre, 1995.

Toffler, Alvin y Heidi. "Mitos del globalismo". La Nación, p. 16A, San José, Costa Rica. Noviembre 1, 1998.

Tünnerman Bernheim, Carlos. Educación Superior de cara al siglo XXI. San José, Costa Rica: Editorial Mirambell, S.A, p. 62. 1999.

. Educación Superior de cara al Siglo XXI. San José: Editorial Mirambell, S.A, p. 62. 1999.

Xabier Gorostiaga S. J. La construcción de las utopías desde la cultura y la educación. Esquema metodológico y proyecto educativo. Conferencia presentada en el Simposium Educar para construir el sueño: ética y conocimiento en la transformación Social. Guadalajara, p. 8. Febrero 23-26, 2000. 